\title{
DNA VACCINATION OF PIGS WITH PLASMIDS EXPRESSING THE NUCLEOPROTEIN OF PRRS VIRUS
}

\author{
Annette Malene Barfoed ${ }^{1}$, Birte Kristensen ${ }^{2}$, Merete Blixenkrone-Møller ${ }^{2}$, \\ Søren Kamstrup ${ }^{1}$ \\ ${ }^{1}$ Danish Veterinary Institute of Virus Research, Lindholm, Kalvehave, Denmark. \\ ${ }^{2}$ Royal Veterinary and Agricultural University, Copenhagen, Denmark.
}

Porcine reproductive and respiratory syndrome (PRRS) is an economically significant viral disease common in swine producing countries all over the world. The clinical parameters comprise late term abortions, stillborn, mummified and weak born piglets and interstitial pneumonia in neonatal and young pigs. In order to develop rational, efficient and safe ways to control the further spreading of the disease, the ability of the individual PRRS virus (PRRSV) proteins to induce protective immune responses must be elucidated. The aim of this project is to optimise DNA immunisation of pigs in order to use this vaccination technique to test the immunological abilities of the individual PRRSV proteins. For this the immunodominant nucleocapsid protein $(\mathrm{N})$ from PRRSV has been used as model protein.

We have compared two different DNA immunisation techniques (intradermal/intramuscular (ID/IM) injection and GeneGun immunisation), and tested the importance of the chosen administration route for GeneGun immunisation, comparing skin and mucosa as immunisation sites. The efficacy of three related plasmid vectors containing various amounts of immunostimulating CpG-sequences has also been investigated. Furthermore, the influence of the vaccinations on post challenge virus specific antibody titers has been followed. DNA immunisation with both techniques generated high N-specific antibody titers. However, the antibody response in the GeneGun immunised pigs arose earlier and resulted in higher titers than in the ID/IM immunised pigs. As administration route the tongue mucosa was observed to be of lower importance, while immunisation on the epidermis of the ear was very efficient. No significant difference in stimulation of the immune response was observed between the three vectors. Post PRRSV challenge control pigs showed a normal anti-viral antibody response, while the vaccinated pigs showed a more rapid anamnestic response with very high anti-viral antibody titers. We therefore conclude that the DNA immunisation technique has been well adapted for use in pigs and we now have a useful model to test the immunological abilities of the remaining PRRSV proteins. 